\title{
Origami interleaved tube cellular materials
}

\author{
Kenneth C Cheung ${ }^{1}$, Tomohiro Tachi ${ }^{2}$, Sam Calisch $^{3}$ and Koryo Miura ${ }^{4}$ \\ ${ }^{1}$ National Aeronautics and Space Administration, Ames Research Center, Moffett Field, CA 94035, USA \\ ${ }^{2}$ Department of General Systems Studies, The University of Tokyo, Komaba 3-8-1, Meugro-Ku, 153-8902 \\ Tokyo, Japan \\ ${ }^{3}$ Massachusetts Institute of Technology, Center for Bits and Atoms, Cambridge, MA 94035, USA \\ ${ }^{4}$ Professor Emeritus, The University of Tokyo, Japan \\ E-mail: kenneth.c.cheung@nasa.gov, tachi@idea.c.u-tokyo.ac.jp, sam.calisch@cba.mit.edu and \\ miurak@gakushikai.jp
}

Received 1 February 2014, revised 21 April 2014

Accepted for publication 24 April 2014

Published 11 August 2014

\begin{abstract}
A novel origami cellular material based on a deployable cellular origami structure is described. The structure is bi-directionally flat-foldable in two orthogonal ( $x$ and $y$ ) directions and is relatively stiff in the third orthogonal $(z)$ direction. While such mechanical orthotropicity is well known in cellular materials with extruded two dimensional geometry, the interleaved tube geometry presented here consists of two orthogonal axes of interleaved tubes with high interfacial surface area and relative volume that changes with fold-state. In addition, the foldability still allows for fabrication by a flat lamination process, similar to methods used for conventional expanded two dimensional cellular materials. This article presents the geometric characteristics of the structure together with corresponding kinematic and mechanical modeling, explaining the orthotropic elastic behavior of the structure with classical dimensional scaling analysis.
\end{abstract}

S Online supplementary data available from stacks.iop.org/sms/23/094012/mmedia

Keywords: rigid origami, metametrial, cellular structure, dimensional scaling analysis

\section{Introduction}

Origami, the art of folding a piece of paper into a threedimensional (3D) form and/or folding it into a flat state, has received much attention in science and engineering, receiving credit for many innovative designs and applications. Two major approaches for applied origami in mechanical engineering are (a) to obtain stiff and lightweight structures that are manufactured by folding flat sheets, such as folded plate shell structures [1], sandwich panels with origami cores [2, 3], and cellular meta-materials [4], and (b) to obtain flexible deployable mechanisms such as microscale devices [5], mesoscale structures such as folded stents [6] and macroscale structures such as deployable solar panels in space [7]. Prior work has demonstrated the ability to fill space to produce any 3D shape with strings composed of very simple folding mechanisms [8], but much of the work on the extensible structural properties achieved through folding has addressed two dimensional foldings such as tesselations. Schenk and Guest [9] study changes in the material properties by folding egg-box and Miura-ori tessellation patterns to suggest the ability to engineer such properties based on folding patterns. Foldable cellular materials have also been proposed [4], based on the symmetry of Miura-ori, to produce rigid origami deployment mechanisms that are flat-foldable; since the structure deploys to a mechanically singular configuration with flat hinges, the deployed structure is not expected to transform in all of the $x, y$, and $z$ directions, once deployed. Since the stiffness of the former folded-sheet types of origami structures is obtained when the boundary is closed to form cylinders or when each part is assembled and adhered to other sheets, the straightforward expectation is that stiffness and flexibility are mutually exclusive properties of origami.

However, recent studies on rigid origami, i.e., plate-andhinge mechanisms, allow us to design deployable and yet stiff structures through the use of geometrically compatible rigid thick materials [10] or foldable cylindrical and composite structures [11, 12]. The proposition of the cellular structure with a rigid-folding mechanism [12] is based on the assembly of rigidly foldable origami tubes that tesselate to fill space by periodic and affine transformations. Since such a cellular structure with plates tend to produce an overconstrained or 

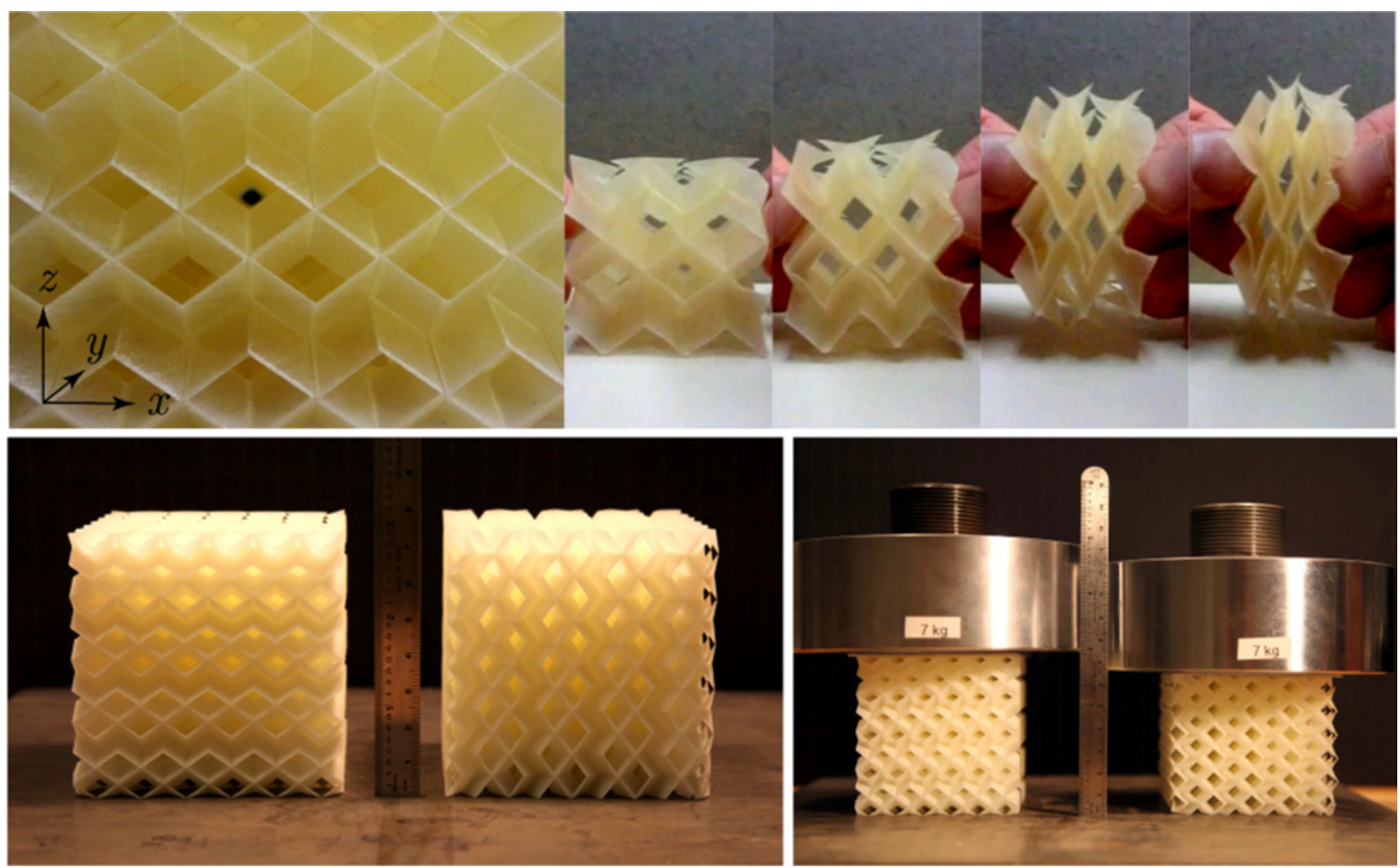

Figure 1. Interleaved tube cellular structure manufactured by 3D printing, showing foldability in $X Y$ direction (top) and rigidity in $z$ direction (bottom). Each of the bottom images shows two copies of the same structure, with the right-hand sample oriented so that the $z$ axis is parallel to the basal plane, and the left-hand sample oriented so that the $z$ axis is perpendicular to the basal plane. Initial conditions are shown in the left-hand set of images, and identical loading conditions are shown in the right-hand set of images, displaying the difference in response for the different orientations.

statically indefinite system, the generic result is a rigid static structure. However, because of the symmetry of the assembly, the overall structure becomes a one degree-of-freedom (DOF) mechanism with desirable robustness and stiffness in its fully deployed state.

In this paper, we propose an anisotropic meta-material based on cellular rigid origami [12]. The material is flexible and stiff at the same time, along orthogonal axes which we name for reference: flexible in the $x$ and $y$ directions and stiff against compression in the $z$-direction (figure 1 ). The interleaved cellular structures are constructed from material with non-negligible thickness, and can be considered to be composed of facets, much like traditional closed-cell materials. While they are strictly not closed-cell structures, because the cells are connected into rows of tubes, rigid configurations of closed faces can contribute significant stiffness through their elemental bending and shearing stiffness. We establish the constitutive volume relationships between the two axes of tubes, and predict the elastic behavior of interleaved tube cellular materials through classical dimensional scaling analysis; specifically, we focus on the material's elastic modulus scaling with relative density. Further, a fabrication technique based on the rigid-foldable deployment mechanism of the structure is proposed, whereby distinct repeating patterns of thin sheets are laminated in a flat state, then deployed to obtain the target $3 \mathrm{D}$ volumetric structure.

\section{Geometry}

\subsection{The unit cell}

The overall geometry and motion of the interleaved tube cellular structure can be represented by its fundamental rhombic dodecahedron unit cell, which is composed of rhombi with sector angles of $\theta_{0}=2 \arctan \frac{1}{\sqrt{2}} \approx 70.53^{\circ}$. We call this module a flip-flop module, since this is an anisotropic scaled version of the kinematic origami known as the 'flip-flop,' with sector angles of $60^{\circ}$, by origami artist Thoki Yenn [13]. A flip-flop module is comprised of two tubular compartments and exhibits continuous flat foldability in the $x$ and $y$ directions. Interestingly, height in the $z$-direction is minimized when it is symmetric in $x$ and $y$ directions, which is also when the volume is maximized (figure 2). This module can tessellate a $3 \mathrm{D}$ space even in folded or partially folded states, because the shearing open rhombic faces are always planar and also parallel to $x z$ and $y z$ planes. Specifically, the configuration can be parameterized by the angles $\theta_{x}$ and $\theta_{y}$ of these open rhombic faces (parallel to $x z$ and $y z$ planes, respectively). These parameters are constrained because the surrounding rhombic faces are filled and maintain rigidity. Normalized direction vectors of the incident edges of a 


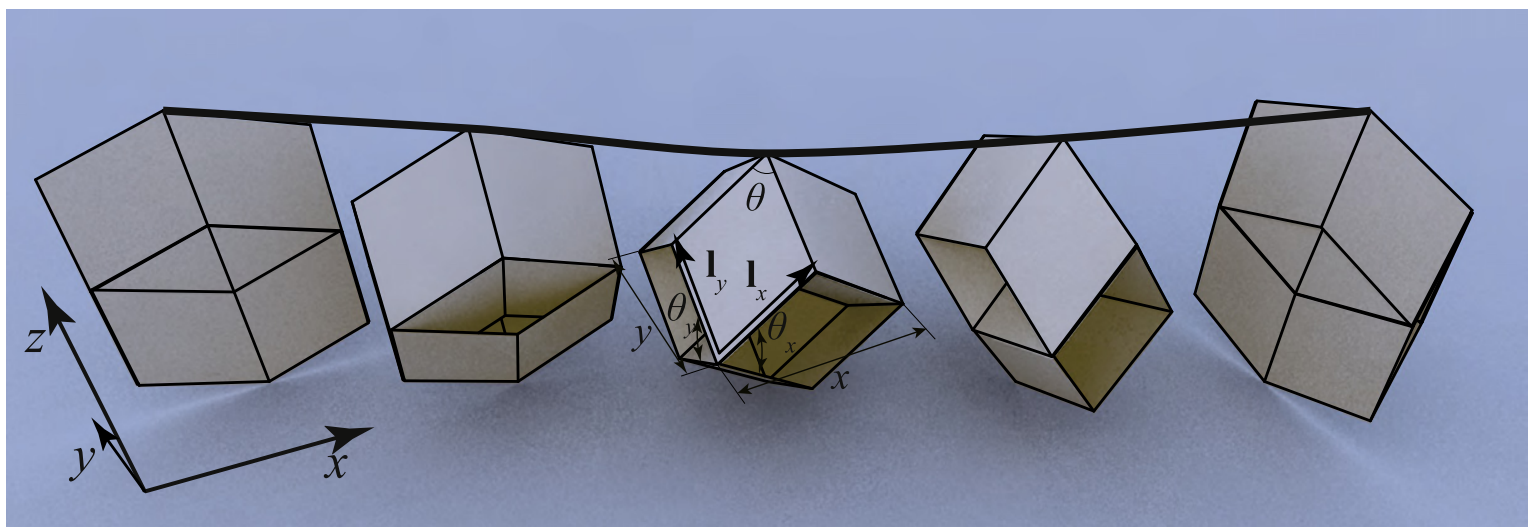

Figure 2. The folding motion of flip-flop. The module flat-folds into $x$ and $y$ directions, while the height $z$ is minimized when the volume is maximized.

rhombus can be given as

$$
\mathbf{l}_{x}=\left[\begin{array}{c}
\cos \frac{\theta_{x}}{2} \\
0 \\
\sin \frac{\theta_{x}}{2}
\end{array}\right] \text { and } \mathbf{l}_{y}=\left[\begin{array}{c}
0 \\
\cos \frac{\theta_{y}}{2} \\
\sin \frac{\theta_{y}}{2}
\end{array}\right] .
$$

Because of the rigidity of the facet, the dot product of these vectors are constant

$$
\sin \frac{\theta_{x}}{2} \sin \frac{\theta_{y}}{2}=\cos \theta_{0}=\frac{1}{3}
$$

and the change in the dimensions are given as

$$
\begin{gathered}
x=2 \ell \cos \frac{\theta_{x}}{2}, \\
y=2 \ell \cos \frac{\theta_{y}}{2}, \\
z=2 \ell \sin \frac{\theta_{x}}{2}+2 \ell \sin \frac{\theta_{y}}{2} .
\end{gathered}
$$

\subsection{Volumetric properties}

The volumes of cells $V_{x}$ and $V_{y}$ along $x$ and $y$ axes, respectively, are

$$
\begin{aligned}
& V_{x}=x \ell^{2} \sin \theta_{y}=4 \ell^{3} \cos \frac{\theta_{x}}{2} \cos \frac{\theta_{y}}{2} \sin \frac{\theta_{y}}{2}, \\
& V_{y}=y \ell^{2} \sin \theta_{x}=4 \ell^{3} \cos \frac{\theta_{x}}{2} \cos \frac{\theta_{y}}{2} \sin \frac{\theta_{x}}{2} .
\end{aligned}
$$

The total volume of the dodecahedron is

$$
V_{x}+V_{y}=\frac{1}{2} x y z=4 \ell^{3} \cos \frac{\theta_{x}}{2} \cos \frac{\theta_{y}}{2}\left(\sin \frac{\theta_{x}}{2}+\sin \frac{\theta_{y}}{2}\right)
$$

Therefore, the following configurations maximize the volume
- $V_{x}+V_{y}$ is maximized to $\frac{16}{3 \sqrt{3}} \ell^{3} \approx 3.079 \ell^{3}$ when $\left(\sin \frac{\theta_{x}}{2}, \sin \frac{\theta_{y}}{2}\right)=\left(\frac{1}{\sqrt{3}}, \frac{1}{\sqrt{3}}\right)$, thus $\theta_{x}=\theta_{y}=\theta_{0} \approx 70.53^{\circ}$, and $x=y=\frac{2 \sqrt{2}}{\sqrt{3}} \ell \approx 1.633 \ell$.

- $V_{x}$ is maximized to $\frac{16}{9} \ell^{3} \approx 1.778 \ell^{3} \quad$ when $\left(\sin \frac{\theta_{x}}{2}, \sin \frac{\theta_{y}}{2}\right)=\left(\frac{1}{\sqrt{5}}, \frac{\sqrt{5}}{3}\right)$ thus $\theta_{x} \approx 53.13^{\circ} \quad$ and $\theta_{y} \approx 96.38^{\circ}, \quad$ and $\quad x=\frac{4}{\sqrt{5}} \ell \approx 1.789 \ell \quad$ and $y=\frac{4}{3} \ell \approx 1.333 \ell$, when the other volume $V_{y}=\frac{16}{15} \ell^{3} \approx 1.067 \ell^{3}$.

- $V_{x}-V_{y}$ is maximized to $\frac{8}{9} \ell^{3} \approx 0.889 \ell^{3}$ when $\left(\sin \frac{\theta_{x}}{2}, \sin \frac{\theta_{y}}{2}\right)=\left(\frac{\sqrt{4+\sqrt{7}}}{3}, \frac{1}{\sqrt{4+\sqrt{7}}}\right)$ thus $\theta_{x} \approx 45.65^{\circ}$ and $\theta_{y} \approx 118.5^{\circ}$, and $x \approx 1.843 \ell$ and $y \approx 1.023 \ell$.

Rigid foldability of these interleaved tube structures combined with this volume relationship theoretically allows for control of fold-state by differential pressurization of the tube volumes, or vice versa. By differentially pressurizing each set of tubes, extension along each of the axes can be prescribed (figure 3) and by varying the total pressurization the overall stiffness can be tuned. To derive the general governing equations we consider pressurizing each of the two sets of cylinders independently and consider the force exerted by the structure in the $x$ direction. For each fundamental flip-flop module, conservation of energy gives:

$$
P_{x} \frac{\partial V_{x}}{\partial \theta_{x}}+P_{y} \frac{\partial V_{y}}{\partial \theta_{x}}=F_{x} \frac{\partial x}{\partial \theta_{x}}
$$

where $P_{x}$ (resp. $P_{y}$ ) is the pressure in the cells of the flip-flop module in the $x$ (resp. $y$ ) direction relative to external pressure, and $V_{x}$ (resp. $V_{y}$ ) is the volume of the cells of the module in the $x$ (resp. $y$ ) direction. $F_{x}$ is the force exerted by the entire module in the $x$ direction. Given that $x=2 \cos \frac{\theta_{x}}{2}$, $\frac{\partial x}{\partial \theta_{x}}=-\sin \frac{\theta_{x}}{2}$. To calculate the volume of one side of the flip-flop module, we use the triple product to compute the volume of a parallelepiped. Defining $\mathbf{l}_{y}{ }^{\prime}$ to be the image of $\mathbf{l}_{y}$ 


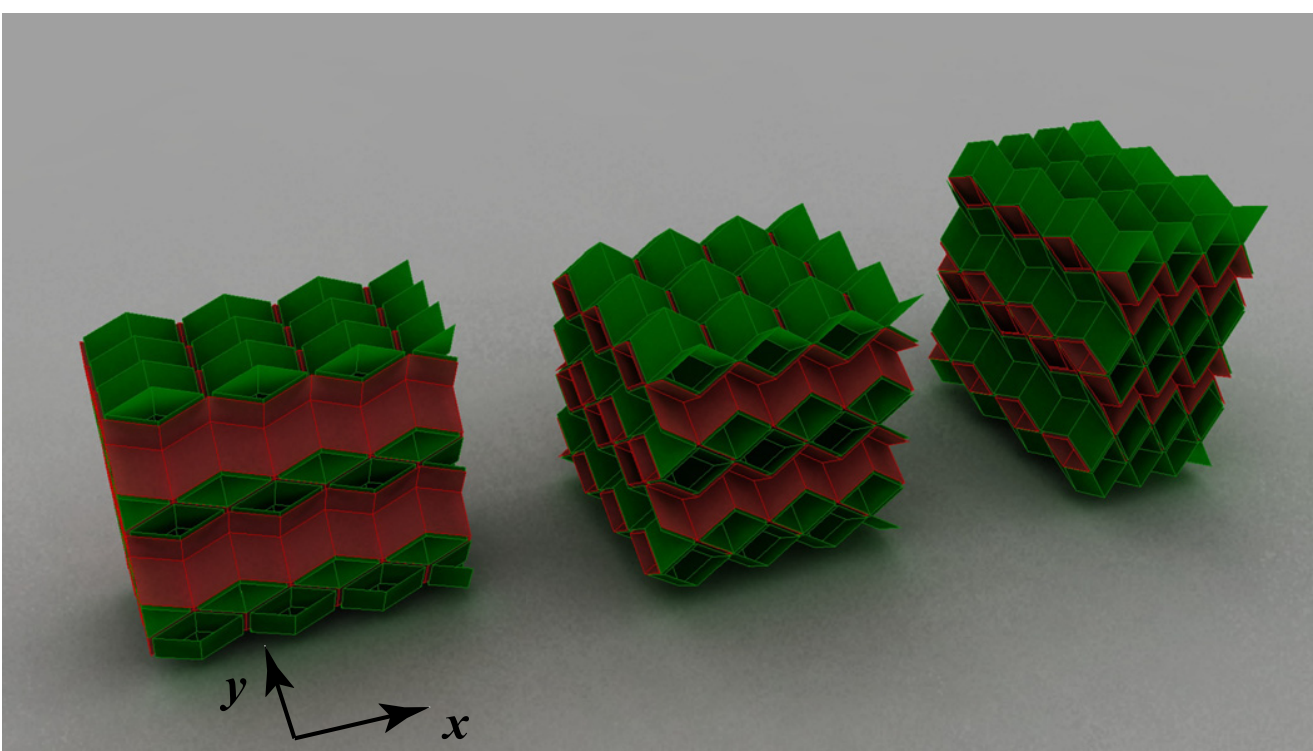

Figure 3. Cellular structure actuated by differentially pressurizing two sets of tubes in $x$ and $y$ directions (colored red and green respectively). Left state maximizes $V_{x}-V_{y}$. Middle state maximizes $V_{x}+V_{y}$. Right state maximizes $V_{y}-V_{x}$.

reflected through the $x y$-plane, we have:

$$
V_{x}=2\left|\mathbf{l}_{y}^{\prime} \cdot\left(\mathbf{l}_{x} \times \mathbf{l}_{y}\right)\right| .
$$

Given that

$$
\begin{aligned}
& \mathbf{l}_{x}=\left(\cos \frac{\theta_{x}}{2}, 0, \sin \frac{\theta_{x}}{2}\right), \\
& \mathbf{l}_{y}=\left(0, \cos \frac{\theta_{y}}{2}, \sin \frac{\theta_{y}}{2}\right), \\
& \mathbf{l}_{y}^{\prime}=\left(0, \cos \frac{\theta_{y}}{2},-\sin \frac{\theta_{y}}{2}\right),
\end{aligned}
$$

for the full chamber (two parallelpipeds),

$$
V_{x}=4 \sin \frac{\theta_{y}}{2} \cos \frac{\theta_{y}}{2} \cos \frac{\theta_{x}}{2} .
$$

Using the rhombus rigidity criterion,

$$
\frac{\partial V_{x}}{\partial \theta_{x}}=-2 \cos \theta_{0} \cos \frac{\theta_{y}}{2},
$$

we can similarly derive

$$
V_{y}=4 \sin \frac{\theta_{x}}{2} \cos \frac{\theta_{x}}{2} \cos \frac{\theta_{y}}{2}, \frac{\partial V_{y}}{\partial \theta_{x}}=2 \cos \theta_{x} \cos \frac{\theta_{y}}{2},
$$

which gives

$$
\begin{aligned}
F_{x} & =-2 \cos \frac{\theta_{y}}{2}\left(\sin \frac{\theta_{x}}{2}\right)^{-1}\left(P_{y} \cos \theta_{x}-P_{x} \cos \theta_{0}\right) \\
& =\cos \theta_{0} \cos \theta_{y}\left(P_{x} \cos \theta_{0}-P_{y} \cos \theta_{x}\right) .
\end{aligned}
$$

If we wish to prescribe a static unloaded configuration, we have $F_{x}=0$. Therefore, to extend to the configuration given to a particular value of $\theta_{x}$, we have the following relation between the pressures:

$$
P_{x}=P_{y} \frac{\cos \theta_{x}}{\cos \theta_{0}} .
$$

This gives a relationship between the two pressures (note that to achieve the regular dodecahedron configuration where $\theta_{x}=\theta_{y}=\theta_{0}$, we have the intuitive result: $P_{x}=P_{y}$ ), but if we want to derive actual values relative to the external pressure, we must specify the desired stiffness of the actuator. This stiffness is given by the derivative:

$$
\begin{aligned}
\frac{\partial F_{x}}{\partial x}= & \left(\frac{\partial F_{x}}{\partial \theta_{x}} / \frac{\partial x}{\partial \theta_{x}}\right) \\
= & \left(-\sin \frac{\theta_{x}}{2}\right)^{-1} \cos \theta_{0}\left(P_{y} \cos \theta_{y} \sin \theta_{x}\right. \\
& \left.+\frac{\partial \cos \theta_{y}}{\partial \theta_{x}}\left(P_{x} \cos \theta_{0}-P_{y} \cos \theta_{x}\right)\right) .
\end{aligned}
$$

In the case of maintaining a static configuration (where $F_{x}=0$ ), this stiffness simplifies to

$$
\frac{\partial F_{x}}{\partial x}=-P_{y} \sin \frac{\theta_{y}}{2} \cos \theta_{y} \sin \theta_{x} .
$$

Equations (9) and (10) dictate the relationship between the pressures, fold-state or configuration, and pressure induced stiffness.

\section{Mechanical model}

In the deployed interleaved tube rhombic dodecahedral structure, the fundamental repeating unit cell may be considered to be the unit rhombic prism with opposing open faces and all faces conforming to the rhombus geometry of the canonical rhombic dodecahedron, which is space-filling by affine transformation. An alternative repeating unit cell for the 


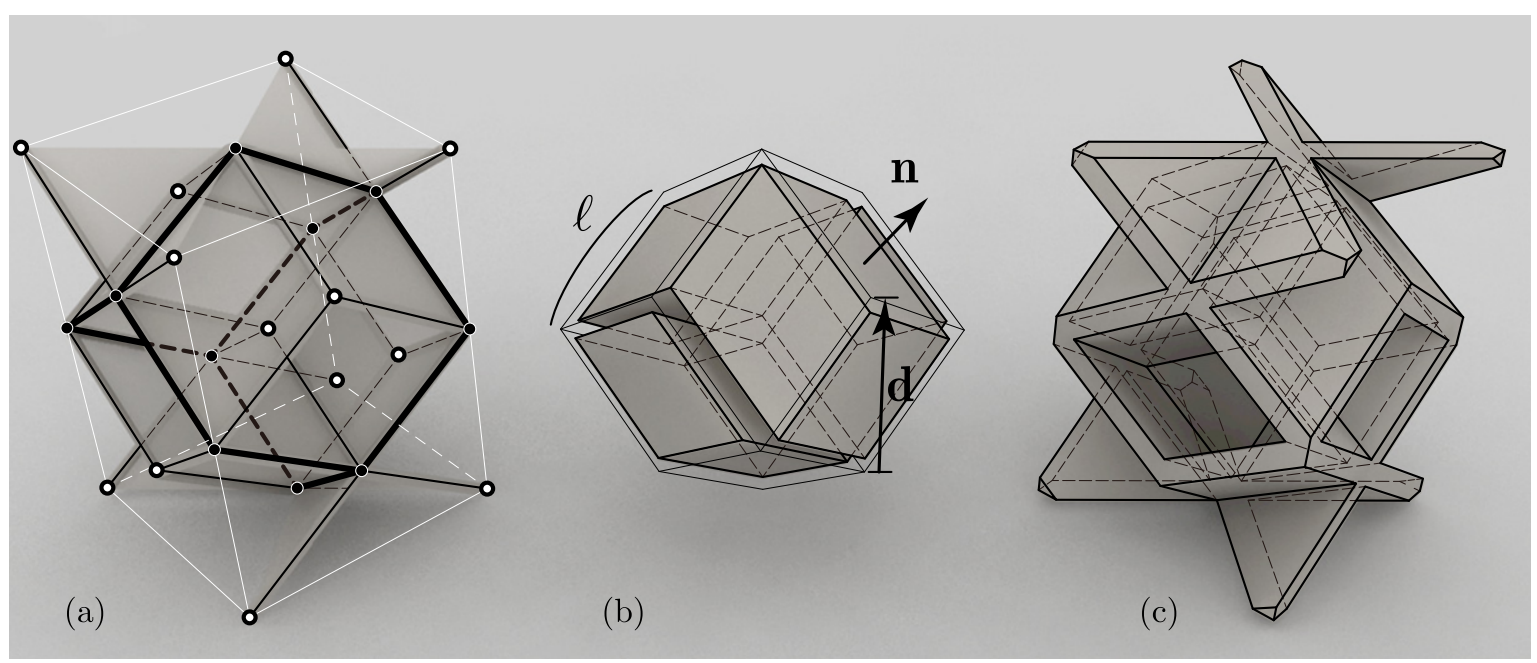

Figure 4. A unit cell of the interleaved tube cellular structure. Left: the white nodes have four incident edges while the black nodes have eight incident edges. The thick lines indicate edges with four incident facets while thin lines indicate edges with two incident facets. Middle: tubular deletions from the theoretical solid, to obtain the structure. Right: thickened cellular structure.

same structure is the rhombic dodecahedron with four open faces and an internal web composed of four rhombic faces, which is space-filling by linear translation. Figure 4 shows another valid repeating unit cell with twice the volume of a single flip-flop, bounded by a rectangular prism with faces parallel to the $X, Y$, and $Z$ planes of the cellular structure. The enclosed rhombic dodecahedron has four open faces and an internal web composed of four rhombic faces, with four additional rhombic faces allowing for space-filling by linear translation on the three orthogonal axes. Considering this webbed rhombic dodecahedron as a composition of two cylinders, the additional rhombic faces that fill out the restangular prism connect the exterior faceted sides of one cylinder to that of its neighboring dodecahedral cell (along the one axis that is orthogonal to both cylinder axes), so as to connect these edges orthogonal to the incident cylinder axis. For simplicity and clarity, we consider this version of this unit cell for the following dimensional scaling analyses. The results of the following analysis are insensitive to the chosen repeating unit cell, provided that the chosen geometry satisfies the requirement of completely tiling space to form the continuous interleaved tube geometry.

The continuous interleaved tube geometry consists of nodes with edge connectivity $z_{e}$ of four and eight in equal proportion (the average edge connectivity $\bar{z}_{e}$ is 6 ), and edges with face connectivity $z_{f}$ of two and four with a number of two connected edges that is twice the number of four connected edges (the average face connectivity $\bar{z}_{f}$ is $\frac{8}{3} \approx 2.7$ ). Assuming high elemental slenderness (thickness « length in both cell edge and face), as is common in the literature, the density scaling relationship in classical form [14] can be simply derived from the geometry as

$$
\frac{\rho}{\rho_{s}} \approx \sqrt{6}\left(\frac{t}{\ell}\right)
$$

where $\rho$ is the mass density of the structure, $\rho_{s}$ is the mass density of the constituent solid material, $t$ is cell edge or face thickness, $\ell$ is cell edge length, and $\sqrt{6} \approx 2.4$ is a geometric constant determined by the deployed interleaved tube rhombic dodecahedral geometry.

Here, we may consider the geometry as if it were created by removing the tubular volumes from a bulk solid (figure 4 (middle and right)). The resulting solid is not strictly rigidfoldable [10], though elastic deformation at the connecting edges allows the structure to deform in a manner that resembles rigid foldability.

If we denote the length of the edge as $\ell$, the height vector of the section rhombus is given by $\mathbf{d}=2 \ell \sin \theta_{0} / 2 \mathbf{e}_{z}$. The scaling factor of each rhombus section of the tube hole is $s=\sqrt{1-\frac{\rho}{\rho_{s}}}$, since each tube hole is obtained by translating the deformed rhombus section along the original zigzagged curve. Then, the relation between face thickness and relative density $\frac{\rho}{\rho_{s}}$, mentioned above, follows as the dot product of the thickness along the height vector and the normal vector $\mathbf{n}$ of a top facet.

$$
t=(1-s) \mathbf{d} \cdot \mathbf{n}=\sqrt{\frac{2}{3}}\left(1-\sqrt{1-\frac{\rho}{\rho}}\right) .
$$

Conventional foams display a lower degree of edge connectivity and higher degree of face connectivity than this deployed interleaved tube rhombic dodecahedral structure. We know from the literature that high edge connectivity is commonly associated with cell edge behavior that is dominated by material stretching instead of transverse beam bending, and associated improvements in specific stiffness scaling, over conventional foams $[14,15]$. The decreased face connectivity is expected from the geometry, and may be seen as an indication of some reduced contribution from cell face/ membrane stiffness, as well as absent contribution from compression of enclosed cell fluid, due to the presence of a fluid path between cells, in this geometry. The small constant 
geometric factor by which relative density scales (with component slenderness $\frac{t}{l}$ ) is similar to conventional foams, and we may consider the interleaved tube rhombic dodecahedral structure to be reasonably space efficient.

The classical closed cell foam specific modulus scaling relationship is known as

$$
\frac{E}{E_{s}} \approx \phi^{2}\left(\frac{\rho}{\rho_{s}}\right)^{2}+p_{0} \frac{1-2 \nu}{E_{s}\left(1-\frac{\rho}{\rho_{s}}\right)}+(1-\phi) \frac{\rho}{\rho_{s}},
$$

where $E$ is the modulus of the foam, $E_{s}$ is the modulus of the constituent solid material, $\phi$ is the fraction of solid material in the cell faces (versus edges), and $\nu$ is the Poisson's ratio of the foam [14]. The three discrete governing phenomena that contribute to this relationship are cell edge bending $\phi^{2}\left(\frac{\rho}{\rho_{s}}\right)^{2}$ [14], compression of enclosed cell fluid $p_{0} \frac{1-2 \nu}{E_{s}\left(1-\left(\frac{\rho}{\rho_{s}}\right)\right)}$ [16], and membrane stretching $(1-\phi) \frac{\rho}{\rho_{s}}$ [17]. The cell edge deflection term omits material stretch, since transverse bending-based deflection will always dominate stretch-based deflection under the same load. If there is enough constraint to limit edge behavior to material stretch, then this term is instead proportional, as $\phi^{2}\left(\frac{\rho}{\rho_{s}}\right)$ [14].

For the case of the interleaved tube cellular structure, as previously mentioned, the cellular chambers are not individually enclosed; however, since the possibility of pressurization and the differential pressure relationship to fold-state may be important for future applications, we include the cell fluid compression term above. We leave future work on pressure dependency to future studies, and focus the remainder of this work on implementations of the material that are not pressure dependent.

Without the cell fluid compression term, considering the open interleaved tube cellular structure alone, we expect the specific modulus scaling relationship to be

$$
\frac{E}{E_{s}} \propto \phi^{2}\left(\frac{\rho}{\rho_{s}}\right)^{2}+(1-\phi) \frac{\rho}{\rho_{s}}
$$

if edge deflection behavior is dominated by microstructural beam bending behavior or

$$
\frac{E}{E_{s}} \propto \phi^{2}\left(\frac{\rho}{\rho_{s}}\right)+(1-\phi) \frac{\rho}{\rho_{s}},
$$

if edge deflection behavior is dominated by material stretch. Regardless of this edge behavior, a minority of the volume fraction of constituent material is in the edges $(\phi \ll 1)$, for the folded interleaved tube cellular structure, so we may expect membrane stretch behavior to yield good specific modulus performance, as

$$
\frac{E}{E_{s}} \propto \frac{\rho}{\rho_{s}}
$$

\section{Results}

To test the above application of classical cellular solids dimensional scaling analysis to the specific modulus scaling of the interleaved tube cellular structure, we performed finiteelement simulations (ELMER software [18]) with finely meshed rigid body models (figure 5). To simplify the implementation of periodic boundary conditions, we chose the repeating unit cell that we introduced in the analytical model above and shown in figure 4.

The simulations predict the material's elastic modulus in accordance with the analytical model. Fitting power law curves to the data yields $E \propto \rho^{1.5}$ in the $z$ axis for a unit cell model with periodic boundary conditions $\left(E \propto \rho^{2.2}\right.$ in the $z$ axis for a unit cell model without periodic boundary conditions), and $E \propto \rho^{2.4}$ in the $x$ and $y$ axes. Performance in these regimes are traditionally explained as expected reductions from analytically idealized scaling laws [15, 19]—in this case, $E \propto \rho$ in the $z$ axis and $E \propto \rho^{2}$ in the $x$ and $y$ axes. This explanation will be addressed further in the discussion section, below.

To evaluate the simulations we performed a brief study of manufactured samples of structures (by stereolithographic 3D printing [20]) and tested their behavior using standard load testing equipment [21] and procedures. The solid constituent material is UV photocured acrylic, fabricated via a stereolithography process that employed a support material with low melting temperature (wax). While the ability to access all surfaces to clear support material was important for this particular process, it is notable that the interleaved tube cellular structure geometry has limited overhang angles for a number of orientations, and we have found that it can therefore be constructed using a variety of commercial layering processes without support material (such as filament fusion/ deposition based processes).

The test specimens were cubic blocks with a 75 millimeter dimension per side, with unit cells approximately 12.5 millimeters in dimension (across the long axis of a rhombic face), and fixtured to face plates on the top and bottom loaded surfaces with high shear strength epoxy (figure 6). It is accepted in the literature that edge effects for cellular materials are minimal beyond characteristic dimensions exceeding several unit cells [22]. We tested four sets of specimens, with mass densities varied by changing the thickness of the cell faces and edges, ranging between $\approx 0.07 \mathrm{~g} \mathrm{~cm}^{-3}$ and $\approx 0.18 \mathrm{~g} \mathrm{~cm}^{-3}$, and corresponding to solid volume fractions of six, eight, twelve, and sixteen percent.

All specimens responded as elastic solids, as expected. Modulus data were calculated from the linear elastic regime, and it was found that the specific modulus scaling fell between canonical stretch and transverse beam bending models ( $E \propto \rho$ and $E \propto \rho^{2}$, respectively), with a best fit power law curve of $E \propto \rho^{1.7}$ in the $Z$ direction, and $E \propto \rho^{2.8}$ in the $X Y$ direction. These regimes are traditionally explained as a moderate reduction in performance from idealized $E \propto \rho$ and $E \propto \rho^{2}$ scaling, respectively, resulting from 

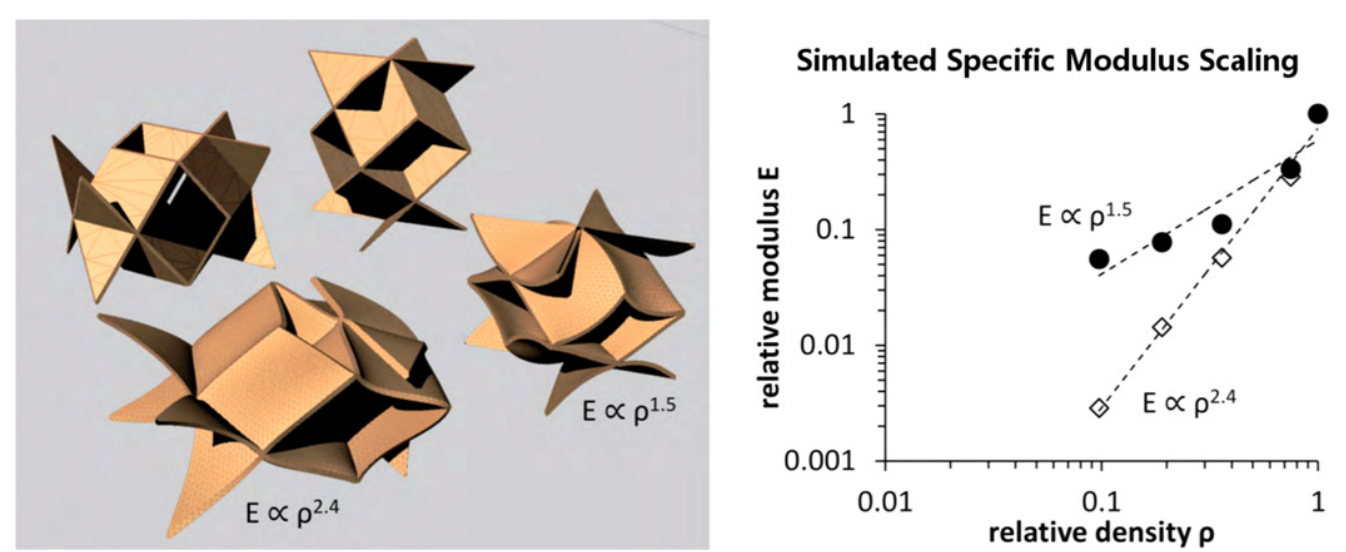

Figure 5. Interleaved tube simulated specific modulus scaling for $X Y$ direction (indicated by open square $\diamond$ ) and $Z$ direction (indicated by solid dot $\bullet$ ). Exaggerated deformations shown, to illustrate deformation mode.

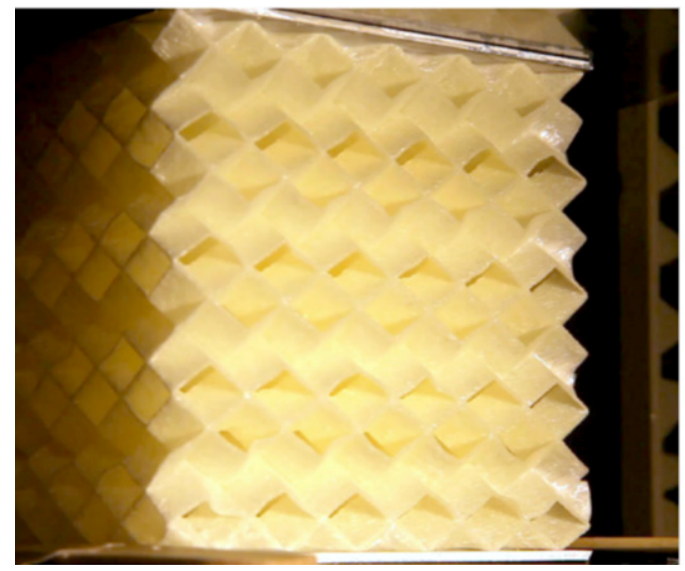

Acrylic Specimen Specific Modulus Scaling

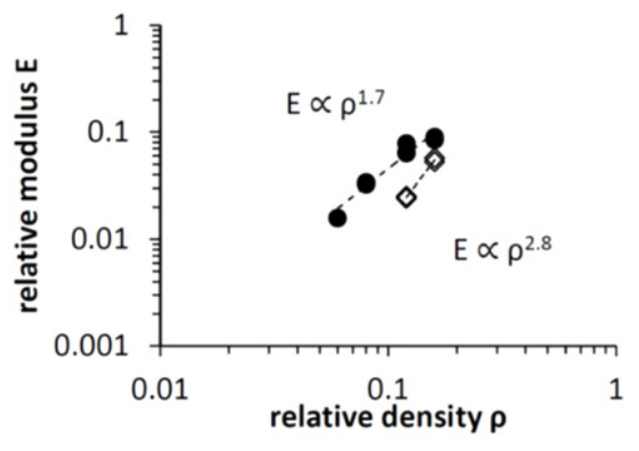

Figure 6. 3D printed acrylic interleaved tube cellular material specific modulus scaling for $X Y$ direction (indicated by open square $\diamond$ ) and $Z$ direction (indicated by solid dot $\bullet$ ), experimental.

manufacturing defects [15] or stochastic variations in geometry that are poorly accounted for by simple periodic models [19]. To explain this difference between the idealized model and experimental results for this particular case, we consider likely contributing phenomena to include microstructural defects and edge effects produced by the stereolithography process (diffusion and mixing between modeling and support polymers), in addition to an adjustment to the idealized model-an alternate stretch-bend coupled model that is presented in the discussion, below.

\section{Fabrication by lamination}

The interleaved tube cellular structure folds to a flat state with multiple overlapping layers, and may be manufactured by sequential lamination of two dimensional patterns with appropriate edge bonding. Reversing the process of flatfolding enables us to then produce the three dimensional cellular structure based on the one-DOF deployment mechanism from the laminated flat state. The approach is similar to the fabrication of a conventional 2.5D honeycomb structure. Since adjacent layers are connected at the edges for the ideal geometric model, we first modify the geometry to have a finite width at the gluing paths, without losing its fundamental mechanism. Figure 7 shows the folding motion of a modified structure that has finite double covered area. Here, each zig-zagged tube structure in one direction is chamfered to produce a thin quadrangle gluing facet that replaces the gluing path. Then, the flat state is produced by laminating four distinct repeating layer patterns shown in figure 8. The patterns shown are comprised of cutting and folding lines and gluing (or sewing) paths to the next layer, and the process can be performed by manual or automated roll-to-roll presses, or CNC controlled cutting and gluing machines. Another efficient implementation of this structure is to use stereo-lithography or 3D printing technology to print the structure in a nearly flat state, noting again that it is trivial to orient the structure so that overhang angles are minimized, allowing for unsupported 3D manufacturing processes, as well.

\section{Discussion}

The typical 3D closed-cell foam structure has pseudo isotropic elastic modulus governed by transverse cell edge bending, resulting in nearly quadratic scaling with respect to 


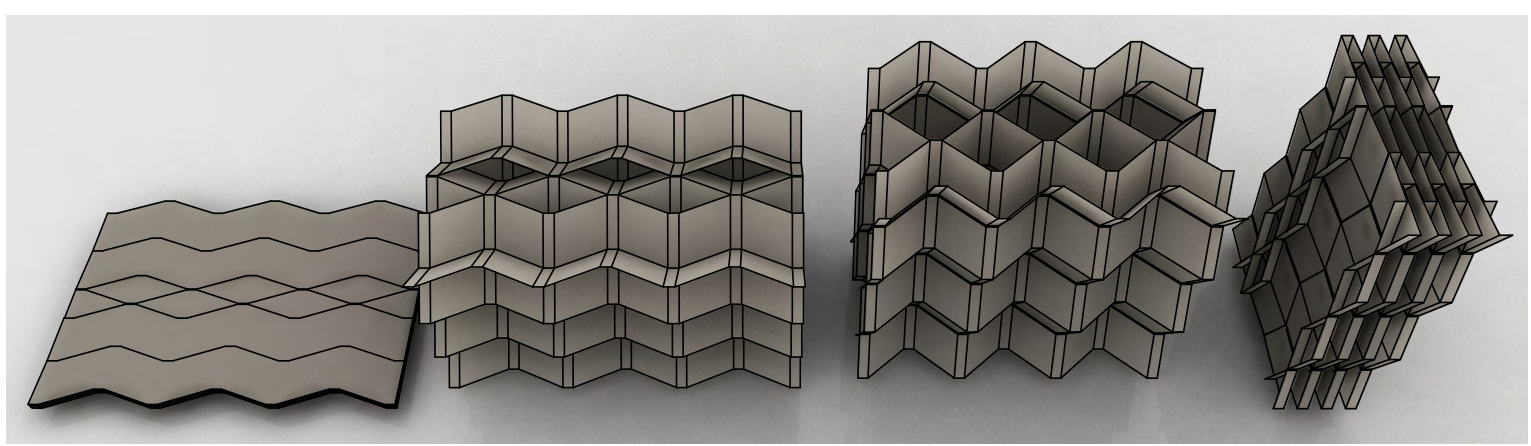

Figure 7. Cellular structure with gluing tabs. The modification maintains the rigid-foldability of the cellular structure.

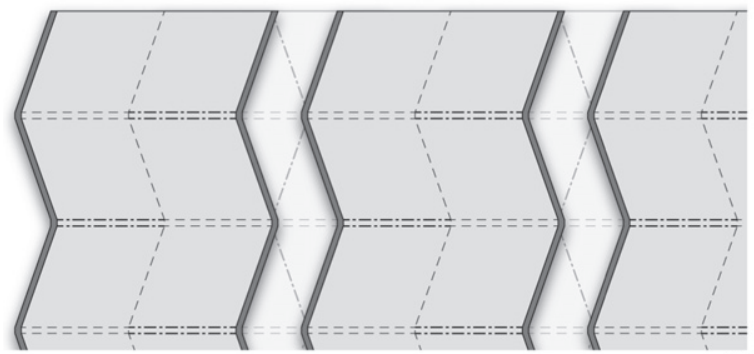

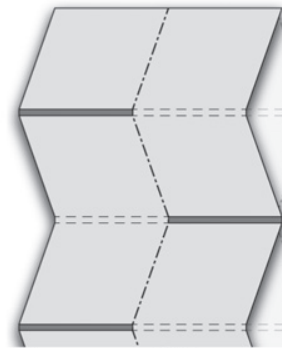

a)

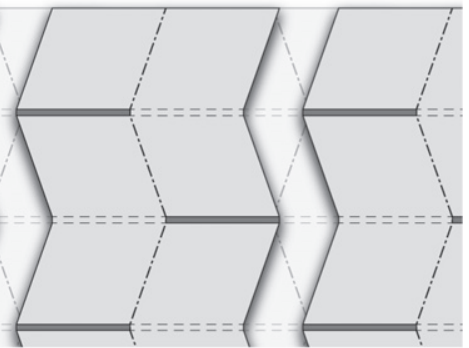

valley

(d) mountain

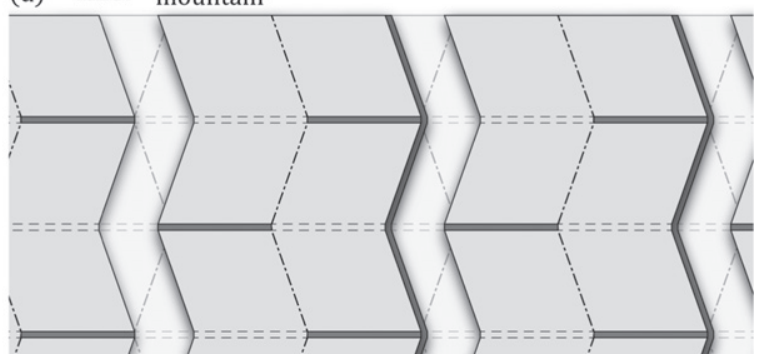

Figure 8. Four cutting-folding-gluing patterns that repeatedly appear from the bottom to top in the order: (a) $\rightarrow$ (b) $\rightarrow$ (c) $\rightarrow$ (d) $\rightarrow$ (a) $\cdots$. Note that the glue is placed on top of the sheet, so that the next layer is welded on the previous layer

its relative density [14]. It is well known that the mechanical properties of such a cellular structure can be improved up to proportional relative elastic modulus, based on the degree of geometric constraint [14] — many such examples of engineered cellular solids exist. From the standpoint of mechanical performance, the interleaved tube cellular materials presented here are shown to approach the mechanical performance of the best known engineered orthotropic cellular materials. Some 2.5D honeycombs, such as typical hexagonal honeycomb core materials, display similarly orthotropic behavior with nearly proportional elastic modulus scaling with density on one axis and significantly reduced modulus scaling in the orthogonal plane [14].

The results here present the material's elastic modulus of order $\approx 1-1.5$ with respect to its relative density in its stiff orientation $(z$ axis) and elastic modulus of order $\approx 2$ in the flexible orientations ( $x$ and $y$ axes).

Prior work has suggested that a bulk behavior model may be derived from a combination of micro-structural component stretch and moment induced component bending, resulting in observed behavior that is dominated by the latter, larger phenomenon [23]. Stretch-bend coupled models have also been applied to biomaterials with mechanical performance that exceeds the predictions of traditional models, based on micro-structural topology [24].

A dimensional scaling argument that is parallel to the classical stretch and transverse component bending models [14], with a bending moment induced component deflection yields a characteristic modulus scaling of $E \propto \rho^{1.5}$. Consider a microstructural element model with a length $\ell$ and thickness $t$, made from a constituent solid material with modulus $E_{s}$, and a bulk cellular material loading condition that results in a specific load $P$ on the component. For a cellular geometry with a high degree of connectivity, we consider component deflection as resulting from both stretch $\left(\delta_{\text {stretch }} \propto \frac{P \ell}{E_{t}{ }^{2}}[25]\right)$ and moment induced bending $\left(\delta_{\text {moment }} \propto \frac{M \ell^{2}}{E_{s} I}\right.$ [25]), with the moment arm being proportional to the element thickness, as opposed to its length as with transverse component bending based deflection. We expect then that the linear response is 
dominated by the deflection due to the bending moment

$$
\delta=\delta_{\text {stretch }}+\delta_{\text {moment }} \propto \frac{P t\left(\ell^{2}\right)}{E_{s} I} \propto \frac{P\left(\ell^{2}\right)}{E_{s} t^{3}},
$$

since $I \propto t^{4}$ [25], and given the conventional assumption that relative modulus is proportional to load and inversely proportional to deflection, length, and $E_{s}$, i.e., $\frac{E}{E_{s}} \propto \frac{P}{\delta E_{s}}$ [14], we expect relative modulus to be proportional to the cube of the component aspect ratio, i.e., $\frac{E}{E_{s}} \propto \frac{t^{3}}{t^{3}}$. Taking the relative density of lattice structures to be proportional to the square of the component aspect ratio, i.e., $\frac{\rho}{\rho_{s}} \propto \frac{t^{2}}{\ell^{2}}$ [14], we find that the expected relative modulus of a cellular material with this mechanism will scale with the relative density by an exponent of $\frac{3}{2}$, i.e., $\frac{E}{E_{s}} \propto\left(\frac{\rho}{\rho_{s}}\right)^{1.5}[23]$.

Whether or not we accept a stretch dominated or stretch-bend coupled model for the specific modulus scaling with density, these interleaved tube cellular solids will likely not be competitive against standard 2.5D honeycomb materials, for identical applications. However, the ability to conveniently access the interleaved enclosed tubular volumes for flow or pressurization may provide interesting application opportunities.

Since the cylindrical chambers can be accessed from the exterior boundary of the structure, active pressurization can be made to dominate the stiffness behavior of the bulk system, and to exceed what is possible through solid material that has not been pre-stressed. Differential pressurization between the two cylinder axes can also be used to drive actuation near the fully deployed state, and differential pressurization between both cylinder axes and the external environment can be used to drive overall deployment actuation. The ability to separate the cell fluid pressurization process from the structure manufacturing process enables this functionality, as well as the ability to make the cell fluid compression term dominate the mechanical behavior of the bulk system.

Interleaved tube cellular structures may also act as a pump to drive the two channels of fluid. For instance, by actuating one of the spatial directions, fluid could be drawn into the structure from both the $x$ and $y$ directions. Besides simply pumping fluids, this could be an effective way to build a heat exchanger due to the large shared surface area between the sets of cylinders. The performance of a heat exchanger is usually measured by its capacity for thermal transmission relative to pressure required to achieve a desired flow rate. There is a precedent for the application of cellular materials to build heat exchangers [26-28] but most of these are single fluid designs. The interleaved tube cellular structure affords complete employment of the constituent membrane material as interfacial surface between the two flow axes. Further, movements of a cellular material have not been previously considered as a mechanisms for optimizing flow conditions.

In addition, a practical layer-by-layer fabrication method of the structure is proposed. The principle is to construct the structure in a flat-folded state with multiple layers by laminating sheets with specified shapes and gluing patterns. Then, the sheets are stretched or inflated to produce a folding motion to deploy the 3D structure. This is potentially applicable to roll-to-roll processes, stereo-lithography and 3D printing, or sheet lamination with $\mathrm{CNC}$ profiling machines (cutting and gluing), so that we can apply the structure at different scales, from microelectromechanical systems to deployable structures for architecture and aerospace.

\section{Acknowledgments}

The authors would like to acknowledge the support of the NASA Ames Research Center, the University of Tokyo, and the MIT Center for Bits and Atoms. The first author's contributions were supported by the NASA STMD Center Innovation Fund. The second author is supported by the JST Presto Program.

\section{References}

[1] Yoshimura Y 1951 On the Mechanism of Buckling of a Circular Cylindrical Shell under Axial Compression The reports of the Institute of Science and Technology University of Tokyo NACA-TM-1390

[2] Miura K 1975 New Structural Form of Sandwich Core J. Aircr. 12 473-441

[3] Klett Y and Drechsler K 2011 Designing Technical Tessellations ed P Wang-Iverston, R Lang and M Yim Origami $^{5}$ : Fifth Int. Conf. on Origami in Science Mathematics and Education (5OSME) (Boca Raton, FL: CRC Press) pp 305-22

[4] Schenk M and Guest S 2013 Geometry of Miura-folded metamaterials Proc. Natl Acad. Sci. 110 3276-81

[5] Bassik N, Stern G M and Gracias D H 2009 Microassembly based on hands free origami with bidirectional curvature Appl. Phys. Lett. 95091901

[6] You Z and Kuribayashi K 2003 A Novel Origami Stent Proc. of the 2003 Summer Bioengineering Conf. pp 257-8

[7] Tanizawa K and Miura K 1978 Large displacement configurations of Bi-Axially compressed infinite plate Trans. Japan Soc. Aeronaut. Sci. 20 177-87

[8] Cheung K C, Demaine E D, Bachrach J R and Griffith S 2011 Programmable assembly with universally foldable strings (moteins) IEEE Trans. Robot. 27 718-29

[9] Schenk M and Guest S 2011 Origami folding: a structural engineering approach ed P Wang-Iverston, R Lang and M Yim Origami ${ }^{5}$ : Fifth Int. Conf. on Origami in Science, Mathematics and Education (5OSME) (Boca Raton, FL: CRC Press) pp 253-63

[10] Tachi T 2011 Rigid-Foldable Thick Origami ed P Wang-Iverston, R Lang and M Yim Origami ${ }^{5}$ : Fifth Int. Conf. on Origami in Science Mathematics and Education (5OSME) (Boca Raton, FL: CRC Press) pp 253-63

[11] Tachi T 2009 One-DOF cylindrical deployable structures with rigid quadrilateral panels Proc. of the IASS Symposium 2009 pp 2295-306

[12] Tachi T and Miura K 2012 Rigid-Foldable cylinders and cells J. Int. Assoc. Shell Spat. Struct. 53 217-26

[13] Yenn T Flip flop (http://erikdemaine.org/thok/flipflop.html)

[14] Gibson L and Ashby M 1999 Cellular Solids: Structure and Properties. Cambridge Solid State Science Series 
(Cambridge: Cambridge University Press) Available from: http://books.google.com/books?id=IySUr5sn4N8C.

[15] Deshpande V and Fleck N 2000 Isotropic constitutive models for metallic foams J. Mech. Phys. Solids 48 1253-83

[16] Gent A and Thomas A 1963 Mechanics of foamed elastic materials Rubber Chem. Technol. 36 597-610

[17] Patel M and Finnie I 1970 Structural features and mechanical properties of rigid cellular plastics J. Mater. 5 909-32

[18] (CSC - IT Center for Science: Elmer (http://csc.fi/english/ pages/elmer)

[19] Roberts A and Garboczi E J 2002 Elastic properties of model random three-dimensional open-cell solids J. Mech. Phys. Solids $\mathbf{5 0} 33-55$

[20] 3d Systems InVision Si2. 3D Systems, (Rock Hill, SC 3d Systems InVision Si2) (http://3dsystems.com)

[21] Instron 4411. Instron, (Norwood, MA: Instron 4411) (http:// instron.us)

[22] Andrews E, Gioux G, Onck P and Gibson L 2001 Size effects in ductile cellular solids. Part II: experimental results Int. J. Mech. Sci. 43 701-13
[23] Cheung K C and Gershenfeld N 2013 Reversibly assembled cellular composite materials Science 341 1219-21

[24] Broedersz C P, Mao X, Lubensky T C and MacKintosh F C 2011 Criticality and isostaticity in fibre networks Nat. Phys. 7 983-8

[25] Timoshenko S P and Gere J M 1961 Theory of Elastic Stability (New York: McGraw-Hill)

[26] Wang B and Cheng G 2006 Design of cellular structure for optimum efficiency of heat dissipation ed M Bendse, $\mathrm{N}$ Olhoff and O Sigmund IUTAM Symposium on Topological Design Optimization of Structures, Machines and Materials (Solid Mechanics and Its Applications) vol 137 (Netherlands: Springer) pp 107-16

[27] Wadley H N and Queheillalt D T 2007 Thermal applications of cellular lattice structures Materials science forum vol 539 (Zurich: Trans Tech Publ) pp 242-7

[28] Kumar V, Manogharan G and Cormier D R 2009 Design of periodic cellular structures for heat exchanger applications Solid Freeform Fabrication Symposium Symposium Proc. (Austin, TX: University of Texas) pp 738-48 